We are most grateful to the Editor, Professor J L Turk, for permission to reprint the following article, from Clinical and Experimental Immunology, $1979,36,1-7$.

\title{
Research in leprosy
}

\section{A REPORT OF A COMMITTEE SET UP BY THE MEDICAL RESEARCH COUNCIL TO STUDY FUTURE PROSPECTS*}

\begin{abstract}
Summary Recommendations for future research in leprosy include (i) cultivation of $M$. leprae in vitro; (ii) genetic control of susceptibility, including twin studies and HLA typing; (iii) precise antigenic analysis of $M$. leprae; (iv) mechanisms involved in the macrophage response to mycobacterial infections; (v) more use of experimental models such as normal mice infected with $M$. lepraemurium; (vi) reassessment of the protection afforded by BCG; (vii) assessment of protection afforded by killed (armadillo) M. leprae vaccine; (viii) pathogenesis of erythema nodosum leprosum including a study of the effect of thalidomide; and (ix) development of in vitro systems for drug sensitivity testing.
\end{abstract}

\section{Introduction}

The committee was set up for a number of reasons. In the first place, the increasingly widespread recognition of dapsone resistance made it clear that the road to the effective control of leprosy was still undefined and certainly a long one. Secondly, the identification of the nine-banded armadillo as an animal host susceptible to $M$. leprae and capable of yielding hitherto unimaginable quantities of bacteria opened up a whole new range of research possibilities. Thirdly, in addition to these biological developments and perhaps in part because of them, the World Health Organization included leprosy in its Special Programme for Research and Training in Tropical Diseases.

*Members: Professor J K G Webb (Chairman) Department of Paediatrics, University of Newcastle; Professor C A Mims, Department of Microbiology, Guy's Hospital, London; Prof essor J L Turk, Department of Pathology, Royal College of Surgeons of England, London. Correspondence: Prof. J L Turk, Department of Pathology, Royal College of Surgeons of England, Lincoln's Inn Fields, London WC2A 3PN. 
Both the Scientific Working Groups on the Immunology (IMMLEP) and Therapy (THELEP) of Leprosy, set up under the Special Programme, have now made expert comprehensive reviews of their respective fields. This review has focused selectively on those areas for research which seem especially important or likely to be productive, and has tried to identify ways in which resources may be used most effectively to complement and support the WHO Special Programme.

\section{Bacteriology}

\section{IN VITRO CULTIVATION OF $M . L E P R A E$}

Renewed attempts to cultivate $M$. leprae in vitro should constitute the most important aspect of research into leprosy at the bacteriological level. Fresh approaches should include modern cell culture techniques. This would give a steady source of bacteria without the need for an uncommon, expensive host such as the armadillo. If a vaccine against leprosy is developed, there will certainly not be enough armadillo-grown bacteria to satisfy world needs. Bacteria produced in vitro would also be more free from tissue components and micro-organisms likely to contaminate animal products, and thus more suitable for research into the antigenic structure of $M$. leprae, and for the development of a vaccine. In vitro cultivation would also greatly simplify the testing of chemotherapeutic agents for activity against $M$. leprae.

The emergence of dapsone resistance means that new drugs must be developed and, if these are to be widely used in those parts of the world where they are most needed, they must be inexpensive. Unfortunately research into the development of new drugs against leprosy is at a low ebb. It is particularly important, therefore, that testing procedures for anti-leprosy drugs are not unduly complicated, time-consuming or expensive. An in vitro system for the growth of $M$. leprae would be the most attractive solution, but other tests for the viability of $M$. leprae could be developed and used to screen test substances for anti-bacterial activity. For instance, the incorporation of tritiated thymidine (or even tritiated DOPA) by freshly isolated dividing bacteria could give a measure of in vitro activity which might be adapted as a test system (Khanolkar et al. , 1978), and the same method can be used to check for the growth of M. leprae in cultivated human macrophages (Krishnaprasad et al., 1977). Other and perhaps simpler tests of bacterial metabolism and viability could be explored.

There is a strong case for the continued study of mycobacteria other than $M$. tuberculosis and $M$. leprae. These other mycobacteria at times infect and sensitize man, either contributing to disease (as suggested in $M$. vaccae in Crohn's disease), or seriously affecting the response to mycobacterial vaccines such as BCG. Such studies seem likely to make an important and practical 
contribution to our understanding of immune responses to tuberculosis and leprosy.

\section{Epidemiology}

\section{GENETIC CONTROL OF SUSCEPTIBILITY}

There have been a number of reports suggesting a genetic determinant of susceptibility to $M$. leprae. Especially important have been studies of the relative susceptibilities of monozygotic twins. The best study is that of Chakravarti \& Vogel (1973) in India, in which among sixty-two monozygotic pairs, thirtyseven $(59.7 \%)$ were concordant for leprosy and thirty-two were also concordant for leprosy type.

Another approach to the search for a genetic factor has been to survey patients for HLA type. The best study so far has been from the Leiden group (de Vries et al., 1976). Initial studies in Surinam were with HLA-A and HLA-B types. Siblings with the same type of leprosy were found to have a significant excess of identical HLA haplotypes, whereas siblings affected with different types of leprosy shared a haplotype less often than expected. This was taken to indicate that susceptibility both to leprosy and the type of disease are controlled by at least two HLA-controlled genes. Recent studies by the same group in Wardha (India) suggest the association of a particular HLA-determinant (DW2) with susceptibility genes for tuberculoid leprosy. In a small study in Ethiopia of HLA-D identity using mixed lymphocyte culture, Stoner et al. (1978) compared seven patients with their lepromatous siblings, suggesting that susceptibility and the development of lepromatous disease are not under HLAHLA-D control.

\section{STUDIES OF TRANSMISSION}

The route by which $M$. leprae enters and infects the body has not yet been established. The likely possibilities are through the skin or nasal mucosa, or by inhalation into the lungs. New evidence is accumulating in support of airborne transmission. Of particular importance is the observation that the average yield of $M$. leprae from a nose blow in a lepromatous patient is as high as $2.8 \times 10^{8}$ bacilli. These bacilli have been shown to be viable by mouse footpad inoculations: they may remain viable for 1-2 days, and occasionally for as long as 7 days (Davey \& Rees, 1974). Evidence for infection through the nasal mucosa has been provided by biopsy studies. In some patients nasal mucosa biopsy has provided the only evidence of leprosy. M. leprae has been found in the nasal mucosa of household contacts; some biopsies have shown evidence of nerve inflammation suggesting local infection with M. leprae (Chacko et al., 1977). 
Further research on transmission is needed. Immunologically deprived mice could be a useful animal model for the study of infection by inhalation (Rees \& McDougall, 1977). Understanding the mode of infection in man is so important to all control programmes that the possibility of primary infection through the nasal mucosa is clearly a field for intensive investigation.

\section{Immunology}

\section{BACKGROUND OF IMMUNOLOGICAL SPECTRUM}

A major breakthrough was achieved by Ridley and Jopling (1966) in the classification of leprosy according to a clinico-pathological spectrum. This classification, which correlated lymphocytic infiltration, the appearance of cells of the mononuclear phagocyte series and bacillary infiltration with Mitsuda skin test reactivity, indicated that the spectrum of disease ranged from a high resistance (tuberculoid: TT), through a number of borderline points (borderline tuberculoid: BT, borderline: BB and borderline lepromatous: BL), to a low resistance (lepromatous: $\mathrm{LL}$ ). It is suggested that the basis for the spectrum was determined by the immunological status of the individual. Moreover, as host resistance to mycobacteria was manifestly due to cell-mediated immunity, it was logical to suppose that the variations in the clinical status of the patient could be correlated with other parameters of cell-mediated immunity. These included delayed hypersensitivity skin tests to extracts of $M$. leprae and the lymphocyte transformation test.

Early results from Godal and his collaborators, working with relatively small patient samples, appear to confirm the spectrum using these tests (Myrvang et al., 1973). However, a number of observations (Bjune et al., 1976) have indicated that although delayed hypersensitivity skin tests and lymphocyte transformation tests may correlate with the allergic reactivity of the patient, they correlate only broadly with host resistance. At two particular points on the spectrum, TT and BT, correlation is with inflammatory response rather than with resistance. Much stronger reactions are found in BT than in TT. This would indicate a dissociation in the antigens responsible for host resistance and those responsible for allergic reactivity. A number of points follow on from this. Firstly, the use of a soluble skin test antigen like PPDtuberculin derived from $M$. leprae, is not a good reagent for assessing host resistance in leprosy. In practice it has been found that even with an armadillogrown antigen, the best skin test reagent to date is one containing all the antigens of $M$. leprae that would give a nodular granuloma in the skin and be read 2-4 weeks after intradermal injection. Secondly, there is at present no suitable in vitro test that can be used to assess host resistance to $M$. leprae. This is 
especially so as the other widely used parameter of cell-mediated immunity, the leucocyte migration inhibition test, is poorly reproducible and difficult to qualify.

\section{ANTIGENIC ANALYSIS OF M. LEPRAE}

M. leprae is poorly antigenic compared with other mycobacteria. Immunization of rabbits with $M$. leprae derived from armadillos results in antibodies against seven antigens only, as compared with BCG which stimulates production of antibodies against seventy components, and $M$. lepraemurium which produces antibodies against thirty to forty antigens. However, if the rabbit serum is concentrated, antibodies can be detected against twenty $M$. leprae antigens. All seven components that are reasonably strong antigens cross-react with the antigens of other mycobacteria, especially BCG. Antibodies against $M$. leprae antigen 7 , which is equivalent to BCG antigen 60 , are found in the sera of tuberculoid as well as lepromatous patients. So far antigenic analysis of $M$. leprae, which began with striking results from Harboe and his group in Oslo (Harboe et al., 1977), has failed to demonstrate any specific antigen of M. leprae that might be associated with host resistance.

There is no doubt that a more precise antigenic analysis of $M$. leprae is of critical importance in the investigation of the clinical status of patients with leprosy. It is particularly important to determine which antigens are responsible for the allergic manifestations of tuberculoid types of leprosy and which are associated with the development of host resistance. Moreover, it is possible that host resistance depends on the development of an immune response against some of the weakest antigens, which might in turn explain why many patients develop the low resistance lepromatous form of the disease.

\section{EXPERIMENTAL MODELS}

Much of the earlier experimental work in leprosy has been with the thymectomized and irradiated mouse, in which host resistance to $M$. leprae has been artificially reduced. Currently, a number of laboratories are working on mouse infection with $M$. lepraemurium, which is a more natural infection in mice and provides a model where immunological mechanisms are intact. Inbred strains of mice can be divided into two groups, high resistance strains (such as C57B1) and low resistance strains (such as $\mathrm{BALB} / \mathrm{c}$ ). In these, there is direct evidence that host resistance is under genetic control. Although $M$. lepraemurium causes systemic rather than cutaneous disease, the pattern of infection in the different strains directly parallels the spectrum in human leprosy.

M. lepraemurium infection of mice has a number of other advantages over $M$. leprae infection. (1) In a natural infection, it is possible to follow the development and loss of cell-mediated immunity using both the delayed 
hypersensitivity and lymphocyte transformation tests. Different types of delayed hypersensitivity, e.g. Jones-Mote reactivity, can also be studied in this model. (2) The mechanism of failure of cell-mediated immunity and host resistance in a mycobacterial infection can be studied with particular reference to the role of suppressor cells and immunoregulatory mechanisms. (3) Owing to the greater potency of $M$. lepraemurium antigens, it should be easier to characterize them and determine which are responsible for the development of host resistance and which are involved in cell-mediated hypersensitivity mechanisms.

\section{ERYTHEMA NODOSUM LEPROSUM ENL}

Whereas it has been considered that the hypersensitivity mechanisms underlying the cutaneous and nerve lesions of tuberculoid and borderline tuberculoid leprosy and reversal reactions are cell-mediated, it has been suggested that ENL could be an immune complex-mediated reaction.

ENL may in fact be two disease states occurring frequently at the same time and developing about 6 months after the onset of chemotherapy. Cutaneous ENL, which incidentally bears no resemblance to erythema nodosum, has features suggestive of an Arthus reaction. These include cutaneous vasculitis, massive infiltration with polymorphonuclear leucocytes and the demonstration of granular deposits of immunoglobin and complement (C3) in the tissues. Systemic manifestations associated with ENL consist of fever, arthritis, uveitis and a transient proteinuria which is distinct from the massive proteinuria of amyloid disease found in advanced cases of leprosy, especially those with recurrent ENL. The uveitis of ENL, and indeed the ocular manifestations of leprosy in general, are important lesions which have been relatively neglected. All these systemic manifestations of ENL may occasionally be dissociated from the cutaneous manifestations.

Numerous attempts have been made to demonstrate changes in serum complement levels in ENL, including total $\mathrm{CH} 50, \mathrm{C} 3$ and Clq binding tests. Most have shown little correlation. Recently, however, Bjorvatn et al. (1976) have demonstrated increased levels of the $\mathrm{C} 3$ breakdown product, $\mathrm{C} 3 \mathrm{~d}$, in the plasma of $70 \%$ of patients with ENL and in only $18 \%$ of patients with lepromatous disease without ENL. This has suggested that in cutaneous ENL the breakdown of C3 is an extravascular event. No other studies of the different parameters of immune complex formation have looked at the cutaneous and the systemic disease separately. One possbility is that changes in circulating complement and the increase in Clq binding activity only occur in systemic forms of the disease. Moreover, there is no evidence that the cutaneous manifestations are due to immune complexes that are known to deposit in areas where the blood vessels are damaged from other causes. It could be that the primary cutaneous lesion is due to the activation of $\mathrm{C} 3$ through the alternative pathway, which results from 
release of mycobacterial polysaccharides when organisms are killed too rapidly by chemotherapy.

A further line of research into ENL that needs consideration is the role of thalidomide in suppressing this reaction. So far, thalidomide has not been shown to affect any allergic reaction in experimental animals, nor does it appear to affect any other allergic type of reactions in man. Further thought should be given to the action of thalidomide in vivo, as elucidation of its action could throw light on some of the pathological and immunological mechanisms underlying ENL.

\section{A R M A DILLOM. LEPRAE VACCINE}

A protocol has been developed by IMMLEP for isolating $M$. leprae from the tissues of infected armadillos by means of enzyme digestion using collagenase, trypsin and chymotrypsin. Proteolytic enzymes are known to destroy protein antigenicity; the present method of extracting $M$. leprae may therefore be reducing the potency of the preparation and have to be modified.

A vaccine may be ineffective in patients with lepromatous leprosy in whom there is an underlying specific immunological defect and whose tissues are loaded with large numbers of live $M$. leprae. However, such a vaccine will be particularly useful in children at special risk, for example those who are considered uninfected but who live in a leprosy endemic area, and especially those resident in a leprosy household. As there is every indication that armadillo $M$. leprae are not attenuated and would be highly infective for man, these organisms could be used for vaccine only if rendered uninfective. The relative efficiencies of dead mycobacteria and attenuated live mycobacteria in increasing host resistance have been intensively studied over 50 years by workers such as Arnold Rich and Sidney Raffel (Turk, 1975). Whereas a live attenuated vaccine like BCG markedly increases host resistance, dead mycobacterial preparations, even in adjuvant, do not appear to be able to increase host resistance although they produce strong delayed hypersensitivity reactions to tuberculin. The production of an attenuated live armidillo $M$. leprae vaccine comparable to BCG could well take many years. A dead vaccine might produce strong allergic reactivity, possibly without protection. Experiments now in progress on the inhibitory effect of a killed armadillo $M$. leprae vaccine on the limited growth of $M$. leprae in the footpads of normal mice are, therefore, of particular interest (Shepard, Walker \& Van Landingham, 1978). Another approach to this problem is to look at the effect of irradiation in abolishing the infectivity of $M$. leprae without reducing its immunizing potential ( $\mathrm{R} \mathrm{J} \mathrm{W} \mathrm{Rees,} \mathrm{personal}$ communication). A suitable protocol for such a study might be developed using $M$. lepraemurium in mice. 
BCG AND LEPROSY : A RE-APPRAISAL

There is considerable cross-reaction between the antigens of $M$. leprae and those of other mycobacteria. So far, it appears that an antigen specific for $M$. leprae has not been characterized. It is thus logical to look more carefully at the possible usefulness of the most widely available live attenuated mycobacterial preparation, BCG vaccine.

Stanford (1977) has compared the protection conferred by BCG against leprosy in Uganda and Burma with the protection against tuberculosis in the UK and the USA (Table 1). He considered that the differences observed could have been due to differences in the mycobacterial in the environment. He then looked at protection against leprosy in Burma and Uganda as a function of the age at which the children had been vaccinated (Table 2 ).

Table 1

\begin{tabular}{lccc}
\hline & $\begin{array}{c}\text { Protection from } \\
\text { tuberculosis } \\
(\%)\end{array}$ & $\begin{array}{c}\text { Protection from } \\
\text { leprosy } \\
(\%)\end{array}$ \\
\hline UK & 78 & Uganda & 80 \\
USA & 14 & Burma & 17 \\
\hline
\end{tabular}

Table 2

\begin{tabular}{ccc}
\hline \multirow{2}{*}{$\begin{array}{c}\text { Age vaccinated } \\
\text { (years) }\end{array}$} & \multicolumn{2}{c}{ Protected (\%) } \\
\cline { 2 - 3 } & Burma & Uganda \\
\hline $0-4$ & 66 & 78 \\
$5-14$ & 25 & 77 \\
\hline
\end{tabular}

Analysed in this way, the data suggested something had happened to children between the ages of 4 and 15 in Burma to change their response to BCG vaccination. He found that $M$. marianum was more common in Burma than in Uganda and that skin test positivity to $M$. marianum antigens reached $30 \%$ at the age of 10 years. He therefore suggests that $M$. marianum is one of the environmental mycobacteria immunizing children in Burma in a way which blocks their ability to respond to BCG, with the increase in host resistance observed elsewhere. In the laboratory it was found that skin testing of mice by the footpad swelling technique with reagents of high specificity, following challenge with various species of mycobacteria, showed that there were two patterns of response, one for species not pathogenic for mice and another for pathogens. Moreover, he has shown that pre-feeding mice with $M$. marianum converts the response to BCG from the non-pathogenic to the pathogenic. $M$. leprae has been found to have a similar effect to M. marianum, so the possibility 
exists that $M$. leprae vaccination, far from increasing host resistance to $M$. leprae, might actually decrease resistance.

It was considered that more attention should be given to the results of BCG vaccination in leprosy and that further trials should be undertaken in which the BCG is administered shortly after birth.

\section{Chemotherapy}

The early hope that dapscne would be an all-sufficient drug for the treatment of leprosy has not been fulfilled for two reasons, drug-resistance and microbial persistence.

Secondary resistance to dapsone has now been recognised clinically in most countries where leprosy is endemic. Although low dosage and irregularity of treatment appear to facilitate the emergence of resistance, this still develops where treatment is regular and dosage adequate (Meade et al., 1973). The duration of treatment before resistance becomes evident, has varied between 5 and 24 years in one series (Pearson, Rees \& Waters, 1975). Resistance has been encountered only in lepromatous (LL) and borderline lepromatous (BL) cases. These clinical observations have been confirmed in the laboratory by mouse diet, compared with levels of $0.0001 \%$ to which all strains of $M$. leprae tested in the past have been fully sensitive (Ellard et al., 1971).

In this context of a world-wide emergent secondary dapsone resistance, primary infections with resistant $M$. leprae are inevitable, and have now been identified in at least two centres where a careful search has been made (Pearson, Haile \& Rees, 1977; Jacobson \& Hastings, 1978).

Quite separate and distinct from the problem presented by dapsone resistance is the phenomenon of microbial persistence. In cases of lepromatous leprosy, $M$. leprae may remain viable in tissues over long periods of time in spite of apparently effective therapy. In one study, fresh tissue biopsies from skin, nerve, striated muscle and smooth muscle (dartos) from lepromatous patients who had received continuous chemotherapy, principally with dapsone, for 10-12.5 years yielded bacilli which multiplied in mouse footpads, and on successful passage were found to be fully sensitive to $0.0001 \%$ dapsone in the mouse diet (Waters et al., 1974). Persisters have also been isolated from sulphone-resistant patients treated for long periods with clofazimine or with rifampicin, either along or in combination with thiambutosine (Rees et al., 1976). Thus, there is as yet no indication that any of the newer mycobactericidal drugs are more effective than dapsone in preventing persistance. Monitoring lepromatous patients for persistence will be an essential feature of all future chemotherapy trial protocols. 
Persistence of bacteria seems to occur largely or entirely in macrophages. Research into the macrophage response has focused on the lysosomal system. Pathogenic mycobacteria, in a way not well understood, often inhibit lysosomal fusion or the fusion of lysosomes with phagosomes. Because none of the drugs so far tried appears to prevent persistence, it is obviously essential that this line of research should continue to be followed.

Support for research in leprosy is currently provided in the UK by the World Health Organization TIR Special Programme, the Medical Reseach Council, The Wellcome Trust and LEPRA.

\section{References}

Bjorvatn B, Barnetson RS, Kronvall GK, Zubler RH Lambert PH (1976) Immune complexes and complement hypercatabolism in patients with leprosy. Clin. Exp. Immunol. 26, 388.

Bjune G, Barnetson RStC, Ridley DS \& Kronvall G (1976) Lymphocyte transformation test in leprosy: correlation of the response with inflammation of lesions. Clin. exp. Immunol. 25, 85.

Chacko CJG, Mathews M, Jesudasan K, Job CK \& Fritschi EP (1977) Nasal mucosa as a possible site of primary involvement in leprosy. Abstracts of a Symposium on 'Recent progress in immunology of leprosy and chronic mycobacterial infection' New Delhi, November 1977.

Chakravarti MR \& Vogel F (1973) A twin study in leprosy. Topics in Human Genetics, 1, 1.

Davey TF \& Rees RJW (1974) The nasal discharge in leprosy: clinical and bacteriological aspects. Leprosy Rev. 45, 121.

De Vries RRP, Fat RFM, Mehra NK, Vaidya MC \& Van Rood JJ (1977) HLA-linked control of the course of an infection with M. leprae. Abstracts of a Symposium on 'Recent progress in immunology of leprosy and chronic mycobacterial infection' New Delhi, November 1977.

Ellard GA, Gammon PT, Rees RJW \& Waters MFR (1971) Studies on the determination of the mycobacterial inhibitory concentration of 4,4'-diaminodiphenyl-sulphone (dapsone) DDS against M. leprae. Leprosy Rev. 42, 101.

Harboe M, Closs O, Bjorvatn B \& Bjune G (1977) Antibodies against BCG antigen 60 in mycobacterial infection. Brit. med. J. ii, 430.

Jacobson RR \& Hastings RC (1978) Primary sulfone resistant leprosy. Int. J. Leprosy, 46, 116.

Khanolkar SR, Ambrose EJ, Chulawala RG \& Bapat CV (1978) Autoradiographic and metabolic studies of Mycobacterium leprae. Leprosy Rev. 49, 187.

Krishnaprasad H, Nath I, Jailkhani BL, Talwar GP, Rattan Singh, Seydel JK \& Mester L (1977) Evaluation of known and potential antileprosy drugs using growth inhibition of M. leprae in cultivated human macrophages. Abstracts of a Symposium on 'Recent progress in immunology of leprosy and chronic mycobacterial infections' New Delhi, November 1977.

Meade TW, Pearson JMH, Rees RJW \& North WRS (1973) The epidemiology of sulphoneresistant leprosy. Int. J. Leprosy, 41, 684.

Myrvang B, Godal T, Ridley DS, Fröland SS \& Song YK (1973) Immune responsiveness to Mycobacterium leprae and other mycobacterial antigens throughout the clinical and histopathological spectrum of leprosy. Clin. exp. Immunol. 14, 541. 
Pearson JMH, Rees RJW \& Waters MFR (1975) Sulphone resistance in leprosy. A review of one hundred proven clinical cases. Lancet, ii, 69.

Pearson JMH, Haile GS \& Rees RJW (1977) Primary dapsone-resistant leprosy. Leprosy Rev. $48,129$.

Rees RJW, Waters MFR, Pearson JMH, Helmy HS \& Laing ABG (1976) Long-term treatment of dapsone-resistant leprosy with rifampicin: clinical and bacteriological studies. Int. $J$. Leprosy, 44, 159.

Rees RJW \& McDougall AC (1977) Airborne infection with Mycobacterium leprae in mice. J. med. Microbiol. 10, 63 .

Ridley DS \& Jopling WH (1966) Classification of leprosy according to immunity. A five group system. Int. J. leprosy, 34, 255.

Shepard CC, Walker LL \& Van Landingham R (1978) Heat stability of Mycobacterium leprae immunogenicity. Infect. Immunity, 22, 87.

Stanford JL (1977) A change in attitude towards a vaccine for leprosy and an account of some experiments in animals. Abstracts of a Symposium on 'Recent progress in immunology of leprosy and chronic mycobacterial infections' New Delhi, November 1977.

Stoner GL, Touw J, Belehu A \& Naafs B (1978) In vitro lymphoproliferative response to Mycobacterium leprae of HLA-D identical siblings of lepromatous leprosy patients. Lancet, ii, 543.

Turk JL (1975) Delayed Hypersensitivity, 2nd edn. North Holland Publishing Company, Amsterdam.

Waters MFR, Rees RJW, McDougall AC \& Weddell AGM (1974) Ten years of dapsone in lepromatous leprosy: clinical, bacteriological and histological assessment and the finding of viable leprosy bacilli. Leprosy Rev. 45, 288. 